\title{
Nonlinear effects in a conceptual multilayer cloud model
}

\author{
U. Wacker \\ Alfred-Wegener-Institut für Polar- und Meeresforschung, 27515 Bremerhaven, Germany \\ Received: 13 October 2005 - Revised: 31 January 2006 - Accepted: 31 January 2006 - Published: 12 April 2006
}

\begin{abstract}
As conceptual model for a cloud a system is considered which is open for condensate mass transport and subject to internal processes such as cloud microphysical transformation and vertical condensate transport. The effects of microphysical processes are represented in parameterized form and the system is divided into two layers to account for the vertical structure. The evolution is mathematically described in terms of four coupled nonlinear ODEs; the prognostic variables are the mass concentrations of cloud water as well as precipitation condensate in each of the layers.

In the absence of vertical velocity the evolution in the lower layer is triggered by the evolution in the upper layer. In the presence of an upwind, the dynamics in both layers is mutually coupled. Depending on the chosen parameter values up to four steady states are found. When varying the parameter upwind velocity, three regimes are distinguished: For week upwind the long-term evolution is steered by the external sources; for stronger upwind the cloud condensate is blown out of the cloud in the final state and does not contribute to formation of precipitation; for intermediate upwind multiple steady state solution branches arise which characterize the transition between those two regimes.
\end{abstract}

\section{Introduction}

Formation and evolution of clouds and precipitation are the consequence of many interacting processes, among them nucleation of condensate particles, their growth, and their transport due to advection and sedimentation within, into and out of the cloud. Hence we can characterize a cloud system as an open system with internal interactions between the condensate particles.

Correspondence to: U. Wacker

(uwacker@awi-bremerhaven.de)
The evolution of cloud systems is mathematically described by a set of thermo-hydrodynamic equations. In numerical simulation models of the atmosphere, cloud microphysical processes are frequently not modelled in detail but their effect on the prognostic variables is treated in parameterized form; for parameterization models see, e.g., Doms and Schättler (1999) and Cotton and Anthes (1989). Cloud microphysical parameterization schemes are necessarily based on many assumptions, e.g., the type of ice particles is prescribed and assumed unchanged during the integration cycle. Since the growth rate of an ice particle depends on its shape, it will not surprise that model results differ in surface precipitation and in particular in cloud water content, when different particle types are assumed (Reinhardt and Wacker, 2004, 2006).

The parameterization equations for the cloud physical transformations and sedimentation processes turn out to contain nonlinear and autocatalytic expressions in the mass concentration of water and ice, respectively. The formal structure of the prognostic equations for the mass concentrations is similar to that of the equations which describe the evolution of mass concentrations of chemical substances due to chemical reactions, such as, e.g., the famous "Brusselator", see e.g., Nicolis and Prigogine (1977) and Ebeling (1976). This formal analogy of the dynamic equations motivated a qualitative analysis of the dynamics of conceptual open nonlinear cloud physical systems (Wacker, 1992, 1995, 1998). Some typical nonlinear effects, such as transcritical and Hopf-bifurcations are found, and in case of several precipitation species also selection effects arise. It turned out in particular, that the prescribed type of precipitating particles (e.g. rain drop, graupel, flat snow flake) is decisive for the order of the transformation rates (whereby the orders are real numbers) and for the dynamic structure of the system. While the system always develops towards a fixed point, if the precipitating particles are assumed to be of spherical-like shape, matters are different if they are chosen as flat ice particles.

Published by Copernicus GmbH on behalf of the European Geosciences Union and the American Geophysical Union. 
In this case, the system's long-term evolution develops into either a point attractor or a periodic attractor, pursuant to the numerical values of parameters controlling the external forcing. A necessary condition for the occurrence of a limit cycle is derived in terms of parameters which carry the information on the selected type of sedimenting particles.

The conceptual cloud model was combined by Palmer (1996) with a one-dimensional dynamic model for the prognostic variables temperature and velocity, wherein the dependency of the variables on height is accounted for in terms of a few spectral modes. The evolution of the condensate fields at different heights is coupled by vertical advection of condensate. This model describes different vertical motion regimes such as turbulent, gravity-wave or laminar flow types. Palmer suggests that the transition between these flow regimes changes the long-term behaviour of the condensate variables. As an example, for a parameter range for which the cloud model alone is in a limit-cycle regime, the transition from laminar flow regime to gravity-wave regime forces the transition of a periodic attractor to a chaotic attractor.

A simplified version of Palmer's model has been recently investigated by Spassova and Nikolov (2005), who analyzed the dynamics of a single-layer model in the variables cloud water and rain water concentrations and vertical velocity. They also found that the attractor can be a fixed point, a limit cycle, or of chaotic type, depending on the selected parameter values.

Another study emphasising the importance of the nonlinearity of cloud physical transformations is that of Jiang and Smith (2003). Based on a conceptual model for orographic precipitation, they identify a threshold point that separates a precipitating and a nonprecipitating state.

While in Palmer (1996) and in Spassova and Nikolov (2005) the character of the attractor is modified due to the interaction with a nonstationary flow field, it is not clear yet, whether the evolution of condensate concentrations within a certain cloud layer can be triggered simply by the evolution in a neighbouring cloud layer due to vertical mass transport by a stationary flow field. Therefore, in this paper a conceptual cloud model for several coupled layers is defined and investigated with regard to its dynamics. The coupling of the evolution in adjacent layers is due to vertical mass transport by sedimentation of precipitation mass and by advection of cloud water mass; advection of precipitation mass is typically neglected since the vertical motion of precipitating particles is mostly dominated by sedimentation. Vertical velocity will be kept constant to avoid nonstationary forcing and is treated as a parameter.

To that end, we extend the zero-dimensional conceptual cloud model as introduced in Wacker $(1992,1998)$ to a onedimensional model. Different to Palmer (1996), the cloud is divided into few discrete vertical layers, here for simplicity only two layers. Since it is known from the zero-dimensional version that a periodic attractor requires the choice of flat ice particles, we shall restrict the following discussion to such particles types. Owing to the focus on the dynamics of the conceptual cloud model, interaction with the velocity and temperature fields are discarded. The dynamics of the coupled two-layer system will be investigated in terms of finding unique or multiple steady states and their stability, and of deriving information on the system's attractor(s). The solution branches and their stability are calculated using a software package provided by C. Völker (personal communication, 1997) ${ }^{1}$ in combination with MATLAB V7.0.1.

The paper is organized as follows: In Sect. 2 the conceptual model for two layers is discussed for the case of onesided coupling due to sedimentation. In Sect. 3 also vertical advection of cloud water by a constant upwind is allowed; hence we have two subsystems with two-sided interactions. Finally the results are summarized and discussed in Sect. 4.

\section{A coupled two-layer model}

We consider a model of a horizontally homogeneous mixed phase cloud with condensate in form of suspended cloud droplets and large, sedimenting particles (rain drops or ice particles), hereafter called precipitating particles. The effect of microphysical processes in clouds is parameterized by a scheme of the Kessler (1969) type. Thereby the ensembles of droplets and precipitating particles are separately characterized by a single time dependent variable, the mass concentrations of cloud water $(C)$ and of precipitating particles $(P)$.

In the following we concentrate on two important cloud physical processes. They are the growth of precipitating particles due to collection of cloud droplets (i.e. accretion in case of rain drops and riming in case of ice particles) and sedimentation of the large particles. The first process represents an internal transformation between the two condensate categories and the second process signifies a mass sink. All other processes, e.g., phase transformations or horizontal and vertical advection, are summarized into a general value $\Phi$ as external source rate.

Adopting the same model assumptions for the parameterization equations as outlined in Wacker $(1992,1998)$, we arrive at the following equations:

$$
\begin{aligned}
& \dot{C}=\Phi_{c}-b C P^{\beta} \\
& \dot{P}=\Phi_{p}+b C P^{\beta}+\frac{1}{\rho} \frac{\partial F_{p}}{\partial z}
\end{aligned}
$$

$\Phi_{c}$ and $\Phi_{p}$ represent external source rates. The second term $b C P^{\beta}$ stands for the riming/accretion rate. This transfer of cloud water mass into precipitation mass is an autocatalytic process and is mathematically described by a nonlinear expression. $\partial F_{p} / \partial z$ denotes the divergence of the downward sedimentation mass flux $F_{p}$.

\footnotetext{
${ }^{1}$ Völker, C.: Continuation and bifurcation problems with MATLAB, unpublished manuscript, personal communication, 1997.
} 
The parameterization equation for the flux reads

$F_{p}=\tilde{d} P^{\delta}$

$\rho^{-1} \partial F_{p} / \partial z$ is discretized for a vertical layer of depth $\Delta z$ as

$\frac{1}{\rho} \frac{\partial F_{p}}{\partial z}=\frac{1}{\rho} \frac{F_{p u}-F_{p l}}{\Delta z}$

with $F_{p u}$ and $F_{p l}$ denoting the flux at the upper and the lower boundaries of the layer under consideration. The flux out of a layer is assumed to depend on the precipitation mass concentration $P$ within that layer.

The coefficients $b$ and $\tilde{d}$, and the exponents $\beta$ and $\delta$ are determined from the parameterization, see Wacker (1992, 1998). They depend on, among others, the chosen type of ice particles.

We now expand the previous conceptual model with regard to a vertical variation of the model variables. To that end we divide the model cloud into two vertical layers, each of depth $\Delta z$. For simplicity, the same particle type is assumed for both layers, so that the model equations (1) hold for the upper (index 1) as well as for the lower (index 2) layer with equal parameter values $b, \tilde{d}, \beta, \delta$. We use the discretization (3) of the flux divergence and set $d=\tilde{d} /(\rho \Delta z)$. Due to mass conservation the sedimentation flux leaving a layer is the flux entering the next layer below. However, no precipitation mass falls into the upper cloud layer. Thus the dynamics of the conceptual two-layer cloud model is represented by the following set of coupled ordinary differential equations:

$$
\begin{aligned}
& \dot{C}_{1}=\Phi_{c 1}-b C_{1} P_{1}^{\beta} \\
& \dot{P}_{1}=\Phi_{p 1}+b C_{1} P_{1}^{\beta}-d P_{1}^{\delta} \\
& \dot{C}_{2}=\Phi_{c 2}-b_{2} C_{2} P_{2}^{\beta} \\
& \dot{P}_{2}=\Phi_{p 2}+b C_{2} P_{2}^{\beta}+d P_{1}^{\delta}-d P_{2}^{\delta}
\end{aligned}
$$

Similarly, the model can be expanded to a multi-layer model.

The source rates $\Phi_{c 1,2}$ and $\Phi_{p 1,2}$ and the precipitation falling out of the lower layer $\left(d P_{2}^{\delta}\right)$ represent interactions with the environment of the two-layer system. The riming rate $b C P^{\beta}$ is an internal nonlinear autocatalytic transformation of the order $\beta+1$; the precipitation falling from the upper into the lower layer is an external sink or source when considering each cloud layer alone, but it signifies an internal transformation of the order $\delta$ in the coupled two-layer system. Due to the one-sided coupling via sedimentation, the dynamics in the upper layer is independent of the existence of the lower layer. Parameters of the system are the source rates $\Phi_{c 1,2}$ and $\Phi_{p 1,2}$, the coefficients $b$ and $d$ and the exponents $\beta$ and $\delta$.
It turns out that the system has a unique steady state given by:

$$
\begin{aligned}
C_{s 1} & =\frac{\Phi_{c 1}}{b}\left(\frac{d}{\Phi_{c 1}+\Phi_{p 1}}\right)^{\beta / \delta} \\
P_{s 1} & =\left(\frac{\Phi_{c 1}+\Phi_{p 1}}{d}\right)^{1 / \delta} \\
C_{s 2} & =\frac{\Phi_{c 2}}{b}\left(\frac{d}{\Phi_{c 1}+\Phi_{c 2}+\Phi_{p 1}+\Phi_{p 2}}\right)^{\beta / \delta} \\
P_{s 2} & =\left(\frac{\Phi_{c 1}+\Phi_{c 2}+\Phi_{p 1}+\Phi_{p 2}}{d}\right)^{1 / \delta}
\end{aligned}
$$

The four eigenvalues $\lambda$ follow as

$\lambda=\frac{1}{2} A_{i} \pm\left[-b d \delta P_{s i}^{\beta+\delta-1}+\frac{1}{4} A_{i}^{2}\right]^{1 / 2} \quad$ for $i=1,2$

with

$A_{i}=b P_{s i}^{\beta}-b \beta C_{s i} P_{s i}^{\beta-1}+d \delta P_{s i}^{\delta-1}$

that gives

$$
\begin{gathered}
A_{1}=\left[(\beta-\delta) \Phi_{c 1}-\delta \Phi_{p 1}\right]\left(\frac{d}{\Phi_{c 1}+\Phi_{p 1}}\right)^{1 / \delta} \\
-b\left(\frac{\Phi_{c 1}+\Phi_{p 1}}{d}\right)^{\beta / \delta} \\
A_{2}=\left[(\beta-\delta) \Phi_{c 2}-\delta\left(\Phi_{p 2}+\Phi_{c 1}+\Phi_{p 1}\right)\right] \\
\quad\left(\frac{d}{\Phi_{c 1}+\Phi_{p 1}+\Phi_{c 2}+\Phi_{p 2}}\right)^{1 / \delta} \\
-b\left(\frac{\Phi_{c 2}+\Phi_{p 2}+\Phi_{c 1}+\Phi_{p 1}}{d}\right)^{\beta / \delta} .
\end{gathered}
$$

A positive real part of an eigenvalue indicates instability of the steady state. Instability of steady state requires at least one of the $A_{i}$ to be positive which can be fulfilled only for $\beta>\delta$. If the particles are assumed to be nearly spherical, as e.g., rain drops and graupel, $\beta<\delta$ holds, and the steady state is always stable irrespective of the values of $\Phi$. If, however, the particles are interpreted as flat ice particles, $\beta>\delta$ holds, and the real part of the eigenvalue may become positive. Then the steady state is either stable or unstable depending on parameter $\Phi$, that is depending on the strength of the external source rates.

In the following, we shall consider only the case in which the precipitating particles are interpreted as flat ice particles with $\beta>\delta$, because this case shows the more interesting dynamical behaviour. Henceforth, the precipitating ice particles will be interpreted as "aggregates of unrimed radiating assemblages of dendrites or dendrites" according to Locatelli and Hobbs (1974) with $\beta=1.406$ and $\delta=1.085, b=2827.2$, and $d=0.02563$ (in SI units), see particle type " 1 " in Wacker (1998). 


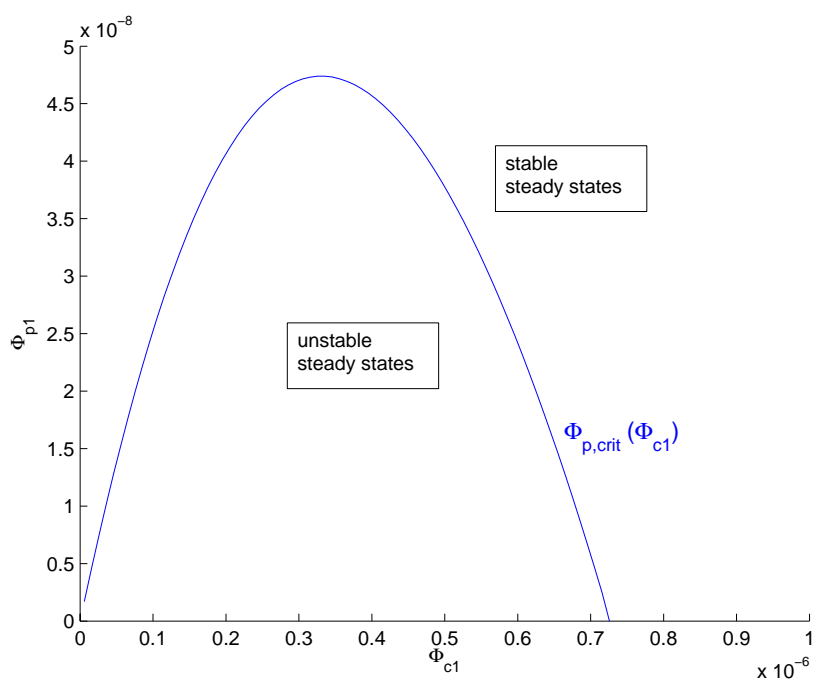

Fig. 1. Critical source rate of $\Phi_{p 1}$ for marginal stability as a function of source rate $\Phi_{c 1}$. Units are $\mathrm{s}^{-1}$.

Marginal stability of the steady state requires parameter combinations such that $A_{i}=0$. We now consider only the upper layer. Figure 1 gives the critical values of $\Phi_{p 1}$, called $\Phi_{p \text {,crit }}$, as function of $\Phi_{c 1}$ at marginal stability. For $\Phi_{p 1}>\Phi_{p, \text { crit }}$ the steady state is stable, otherwise unstable.

For combinations of the parameter values $\Phi_{c 1}$ and $\Phi_{p 1}$ outside the critical curve in Fig. 1 the system will always evolve into a stable steady state, which is a focus (for parameter values in the vicinity of the critical curve) or a node. Below the critical curve $\Phi_{p \text {, crit }}\left(\Phi_{c 1}\right)$ the steady state turns out to be an unstable focus or node and the attractor is periodic. For vanishing external source rate $\Phi_{p 1}$ of the precipitating species, however, it may occur for weak forcing $\Phi_{c 1}$ that $P_{1}$ approaches zero, so that cloud water can be depleted by no mechanism. Such a system turns out unphysical.

Stability or instability of a steady state in the lower layer can be also depicted immediately from Fig. 1, if $\Phi_{c 1}$ is replaced by $\Phi_{c 2}$ and $\Phi_{p 1}$ replaced by the total source $\Phi_{p 2}+d P_{1 s}^{\delta}$ for the lower layer. Obviously, the additional forcing $d P_{1 s}^{\delta}$ owing to the influx from the upper layer tends to stabilize the steady state in the lower layer.

A systematic investigation of the dynamics of the coupled two-layer system distinguishes four cases. They are sorted according to the stability of the steady states for the uncoupled systems:

(1) Stable steady states in both layers:

The $\Phi$-parameters required for this situation are such that the coupled system will always evolve into a stable steady state. (2) Stable steady state in upper layer, unstable steady state in lower layer:

For the coupled layers the steady state sedimentation flux $d P_{1 s}^{\delta}=\Phi_{c 1}+\Phi_{p 1}$ increases the forcing of layer 2 . The steady state is unstable provided $\Phi_{p 2}+\Phi_{c 1}+\Phi_{p 1}<\Phi_{p \text {, crit }}\left(\Phi_{c 2}\right)$.
This combination is possible, however, only for a weak source rate $\Phi_{c 1}$.

If, however, $\Phi_{p 2}+\Phi_{c 1}+\Phi_{p 1}>\Phi_{p \text {, crit }}\left(\Phi_{c 2}\right)$, the impact of sedimentation into the lower layer forces its evolution into a stable steady state.

(3) Unstable steady state in upper layer, stable steady state in lower layer:

The periodic sedimentation flux out of the upper layer acts as periodic forcing on the lower layer and synchronizes a limit cycle in $C_{2}$ and $P_{2}$. In case of a focus for the lower layer with short period, the forced periodic evolution is superimposed by damped oscillation.

(4) Unstable steady states in both layers:

The periodic forcing by periodic inflow of precipitation from above triggers off a periodic evolution in the lower layer, and the entire system develops into a periodic attractor. If the period of the limit cycle in the upper layer is shorter than the period for the stand-alone limit cycle in the lower layer, then $C_{2}$ and $P_{2}$ oscillate in the coupled system with the forcing period. If the period in the upper layer, however, is larger, then $C_{2}$ and $P_{2}$ show the fast oscillation superimposed by the forced slow oscillation. An example for this constellation is shown in Figs. 2 and 3. Time integration is done using the MATLAB routine "ode23s". Figure 2 gives the trajectory in three-dimensional phase space for various combinations of the four variables $C_{1}, P_{1}, C_{2}, P_{2}$, and Fig. 3 shows the evolution of the variables at later times after reaching the periodic attractor. The limit cycle in $C_{1}$ and $P_{1}$ is passed through within a period of about $5000 \mathrm{~s}$. $C_{1}(t)$ has a saw-tooth-like shape. $P_{1}$ is low during most of the time. It increases rapidly after $C_{1}$ has exceeded a certain threshold value due to the autocatalytic riming. The accompanying depletion of $C_{1}$ in return decelerates further increase of $P_{1}$, which is then diminished rapidly due to sedimentation. The periodic behaviour of $C_{2}$ and $P_{2}$ with a period of some $1000 \mathrm{~s}$ is superimposed by the disturbance due to the temporary, but nevertheless periodic forcing from the upper layer. Hence, the dynamics in the lower layer is characterized by the evolution on two different time scales.

\section{The two-layer model with advection}

\subsection{Model equations}

In this section the above model is extended by the effect of vertical advection of cloud water content, $-w \partial C / \partial z$. This additional process changes the two-layer model into a twosided coupled one.

We assume, in contrast to the model of Palmer (1996), a vertical velocity which is constant in space and time. Furthermore we assume a discretization of the advection term of the upstream type, and no cloud water outside the two cloud 

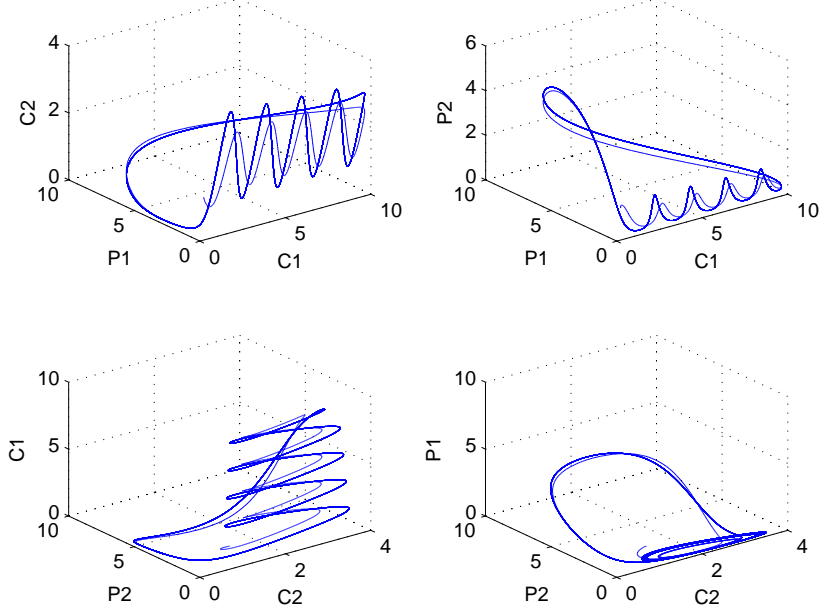

Fig. 2. Trajectories in three-dimensional phase space for case 4 . Plotted mass concentration values are multiplied by $10^{4}$. External source rates: $\Phi_{c 1}=2 \times 10^{-7} \mathrm{~s}^{-1}, \Phi_{p 1}=10^{-9} \mathrm{~s}^{-1}, \Phi_{c 2}=7 \times 10^{-7}$ $\mathrm{s}^{-1}, \Phi_{p 2}=0, \Delta z=100 \mathrm{~m}$. Initial conditions: $C_{1}, P_{1}, C_{2}, P_{2}=10^{-4}$.

layers. We confine here to upwinds $w>0$. Thus the set of Eqs. (4) is enlarged as follows:

$$
\begin{array}{lr}
\dot{C}_{1}=\Phi_{c 1}-b C_{1} P_{1}^{\beta} & -\frac{w}{\Delta z} C_{1}+\frac{w}{\Delta z} C_{2} \\
\dot{P}_{1}=\Phi_{p 1}+b C_{1} P_{1}^{\beta}-d P_{1}^{\delta} & -\frac{w}{\Delta z} C_{2} \\
\dot{C}_{2}=\Phi_{c 2}-b C_{2} P_{2}^{\beta} & \\
\dot{P}_{2}=\Phi_{p 2}+b C_{2} P_{2}^{\beta}+d P_{1}^{\delta}-d P_{2}^{\delta} &
\end{array}
$$

Due to upward motion, the dynamics in the upper layer is affected by the dynamics in the lower. Moreover, due to the absence of cloud water outside the layers, the effect of advection signifies an additional mass sink $-w / \Delta z C_{1}$ for the total system.

A special feature caused by the coupling is discussed now. Figures 2 and 3 show that the evolution in the lower layer is modified by the time-dependent precipitation flux from the upper layer, that is by superposition of a long-term oscillation due to the limit cycle in the $C_{1}, P_{1}$ variables. Figures 4 and 5 show the corresponding trajectories and time series as resulting under the same conditions, but with advection of cloud water mass by an upwind of $w=10 \mathrm{~cm} \mathrm{~s}^{-1}$. It is noted that $P_{1}$ is very low but nonzero. The striking difference to the $w=0$ case is that the long-term evolution is characterized by a periodic oscillation in all variables with the same period of about $3000 \mathrm{~s}$. This period is an intermediate value between the periods charcteristic for $C_{1}, P_{1}$ and $C_{2}, P_{2}$, respectively, in the case $w=0$ (Fig. 3). Due to advection the evolution of $C_{1}$ and $P_{1}$ of the upper layer is triggered off by the periodic cloud water mass transport into the upper layer, which prevents the formation of the long period limit cycles as seen in Fig. 3. The choice of other parameter values may result in

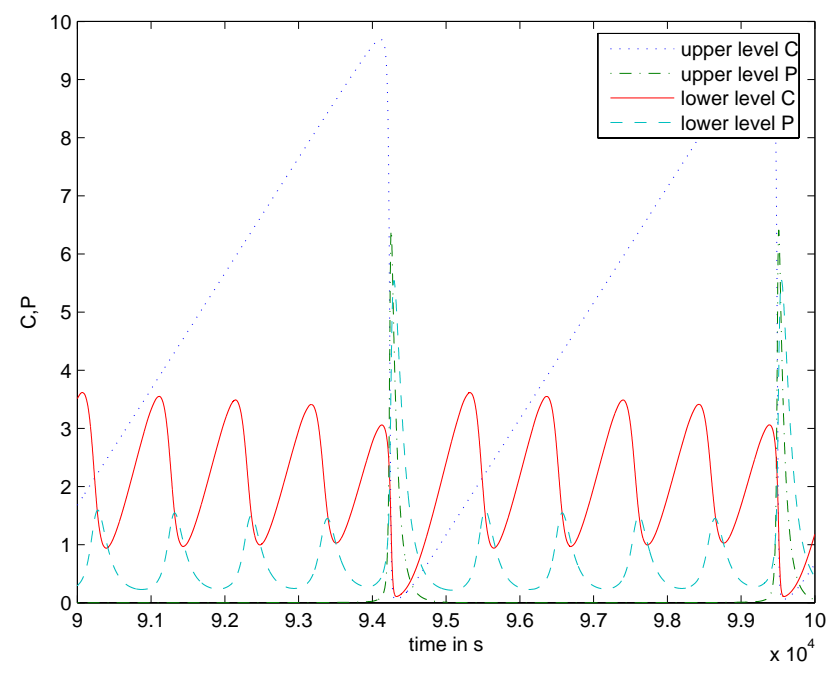

Fig. 3. Time series of cloud water and precipitation mass concentrations (multiplied by $10^{4}$ ) for a time interval 90000 to $100000 \mathrm{~s}$, i.e. after reaching the periodic attractor. Model conditions as for Fig. 2.

a different period of the coupled system or in a steady state attractor.

It is noteworthy that the timescales of the evolution of the concentrations for the examples shown in Figs. 3 and 5 are roughly of the order of an hour. They certainly depend on the control parameters, but they are of the order of time scales known for real clouds. Moreover, Rauber et al. (1986) report from the observation of a cloud system a sequel of two maxima of liquid water content and of precipitation rate; the highest liquid water content occurred prior to the onset of precipitation, and after the onset, liquid water content decreased substantially. This type of evolution is similar to that seen in Figs. 3 and 5. A comparison between the results of the idealised study with real world phenomena is subject to handicaps due to the many model simplifications. Hence, it cannot be decided whether the similar features are due to the same physical mechanisms, or triggered off by other processes as, e.g., nonstationary vertical motion.

\subsection{Special case: $\Phi_{p 1}=0, \Phi_{p 2}=0$}

The special case of no external sources of precipitation mass, that is $\Phi_{p 1}=0$ and $\Phi_{p 2}=0$, will be discussed first. This case is of physical relevance, since the dominant external source and sink for precipitation mass in a cloud layer are the sedimentation mass fluxes into and out of the layer, represented in terms of $d P_{i}^{\delta}$.

This model system turns out to have multiple steady states. One of them is characterized by vanishing precipitation mass concentrations, in which the external sources $\Phi_{c 1,2}$ for cloud 

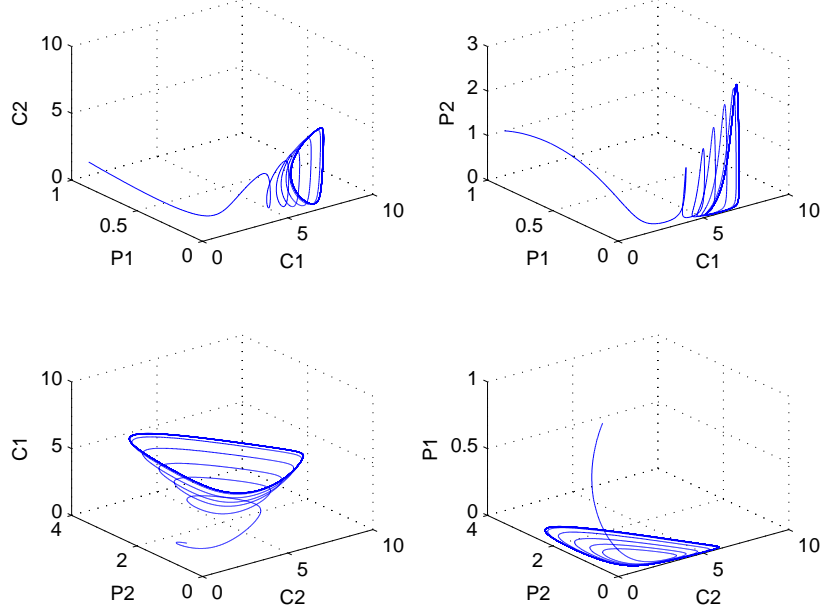

Fig. 4. As Fig. 2, but for vertical velocity $w=10 \mathrm{~cm} \mathrm{~s}^{-1}$.

water are balanced by the advective outflow, that is:

$$
\begin{aligned}
C_{1 s} & =\left(\Phi_{c 1}+\Phi_{c 2}\right) \frac{\Delta z}{w} \\
P_{1 s} & =0 \\
C_{2 s} & =\Phi_{c 2} \frac{\Delta z}{w} \\
P_{2 s} & =0
\end{aligned}
$$

This steady state (11) has no counterpart in the case $w=0$, see previous Sect. 2. A linear stability analysis of the steady state (11) gives two negative and two zero eigenvalues. Integration of (10) shows that the steady state is a point attractor, however, with only a small attracting basin.

Other steady states exist, but are not found analytically. The bifurcation diagram is given in Fig. 6 for fixed $\Phi_{c 2}=10^{-6} \mathrm{~s}^{-1}$ and varying $\Phi_{c 1}$. Multiple steady states are found for all $\Phi_{c 1}$-values. While for weak external forcing three steady states are found for equal parameter values, two further steady states are born at a critical source rate of $2.94 \times 10^{-7} \mathrm{~s}^{-1}$. Beyond the tangent bifurcation, five steady states are found. The numerical differences in some steady state branches are within drafting accuracy. The steady state values of $P_{1}$ on branches 3,4 , and 5 and of $P_{2}$ on branch 4 are zero. It turns out that the steady states are linearly stable or unstable as indicated in Fig. 6 by the solid and dashed lines, respectively. On branch 1 a Hopf bifurcation occurs at $\Phi_{c 1}=7.2783 \times 10^{-7} \mathrm{~s}^{-1}$. Indeed, numerical integration of the set of Eqs. (10) shows that at least for $\Phi_{c 1}$ slightly less than that value, the system possesses a periodic attractor; its attracting basin, however, is limited by the attractor basin of the point attractor on solution branch 4 and eventually vanishes for $\Phi_{c 1}$ smaller than a certain value.

\subsection{General case for $\Phi_{p 1}, \Phi_{p 2} \geq 0$}

In this subsection we shall see how the multiple steady states are modified by additional external sources $\Phi_{p}>0$. Ob-

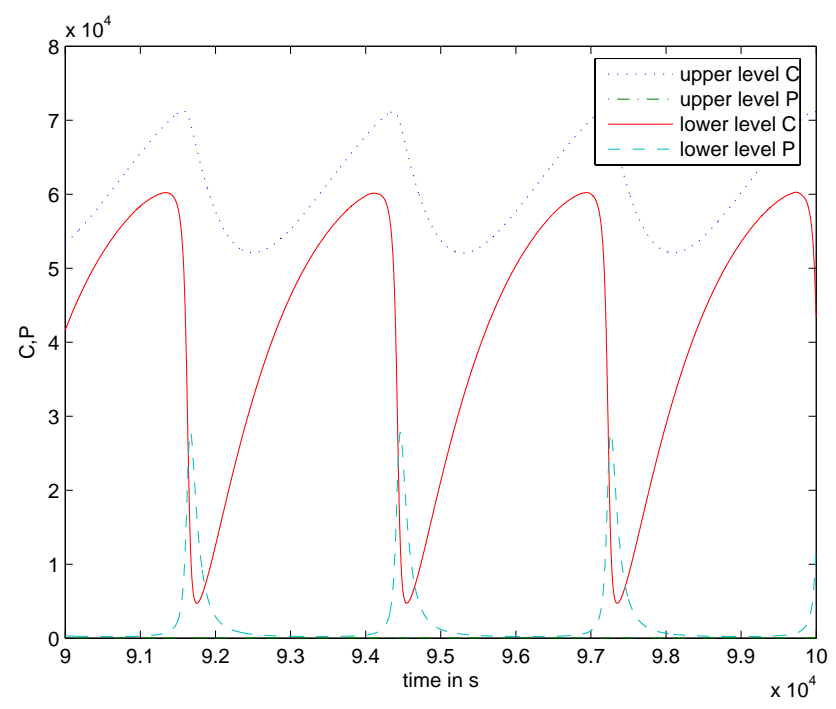

Fig. 5. As Fig. 3, but for vertical velocity $w=10 \mathrm{~cm} \mathrm{~s}^{-1}$.

viously, non-vanishing source rates $\Phi_{p 1}$ or $\Phi_{p 2}$ prevent a steady state with vanishing mass concentration $P_{1}$ or $P_{2}$ as for the branches 3, 4, and 5 in Fig. 6, and it is expected that steady state branches may exist only for parts of the parameter range. Figure 7 gives the bifurcation diagram for the steady states as functions of $\Phi_{c 1}$ for fixed parameters $\Phi_{c 2}=10^{-6} \mathrm{~s}^{-1}$ and $w=10 \mathrm{~cm} \mathrm{~s}^{-1}$, as in Fig. 6, except for $\Phi_{p 1}=\Phi_{p 2}=10^{-9} \mathrm{~s}^{-1}$. Even for this weak source rate for precipitation mass we find qualitative differences in the steady states, and this example illustrates how the solution branches are modified with increasing $\Phi_{p 1,2}$.

Firstly, it is noted that only up to three solution branches are found. Their numbering in Fig. 7 follows that in Fig. 6. Solution branches 4 and 5 with small values of $P_{1}$ and $P_{2}$ exist only up to a certain $\Phi_{c 1}$ value, which in turn decreases with increasing $\Phi_{p 1,2}$. The selected $\Phi_{p 1,2}$-values are such that branches 4 and 5 do not exist any more. Branches 2 and 3 also end at an upper $\Phi_{c 1}$-limit and merge in a cusp, as seen in Fig. 7. This $\Phi_{c 1}$-limit decreases with increasing $\Phi_{p 1,2}$, and for $\Phi_{p 1}=\Phi_{p 2}=10^{-8} \mathrm{~s}^{-1}$ the cusp has already dissappeared; irrespective of $\Phi_{c 1}$ only a unique steady state remains.

In general, increasing external forcing by $\Phi_{p 1,2}$ removes some freedom from the system's dynamics and forces its development into a unique attractor. For the parameter values chosen for the calculations shown in Fig. 7, three steady states are found in a small range of parameter values $\Phi_{c 1}$. This range, however, does neither include very weak nor strong forcing $\Phi_{c 1}$. The attractor turns out to be unique for all $\Phi_{c 1}$-values, that is either the stable steady state on branch 3 , or the stable steady state on branch 1 , or a limit cycle related to the unstable steady state on branch 1 if branch 3 does not exist. Multistability may occur for smaller $\Phi_{p 1,2 \text {-values, }}$ when the branches 3 and 1 with stable steady states coexist

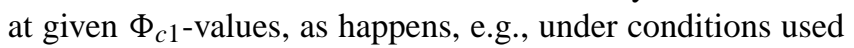
for preparing Fig. 6. 

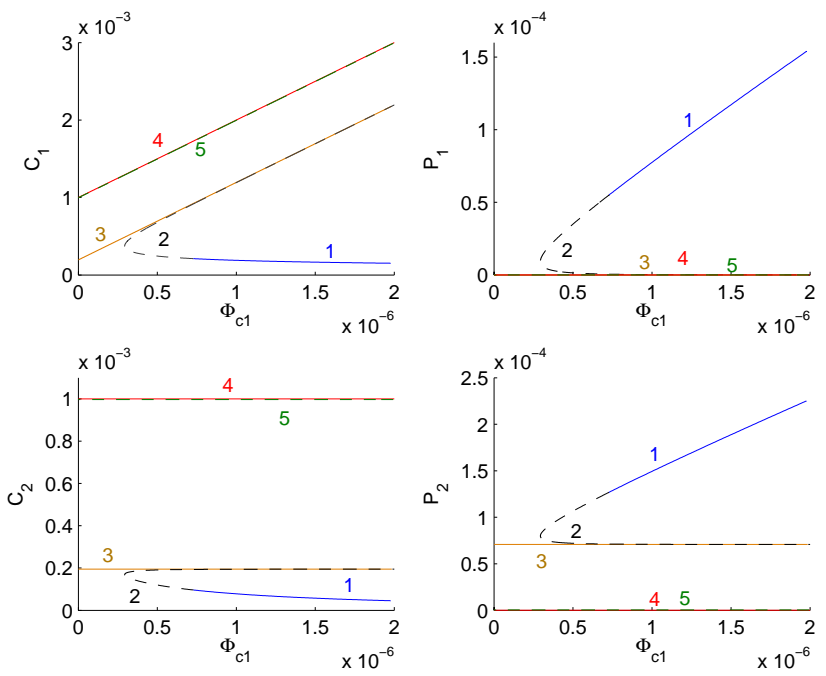

Fig. 6. Bifurcation diagram for steady states as functions of the parameter $\Phi_{c 1}$ for $\Phi_{c 2}=10^{-6} \mathrm{~s}^{-1}, \Phi_{p 1}=0, \Phi_{p 2}=0$, and $w=10 \mathrm{~cm}$ $\mathrm{s}^{-1}$. The five branches are numbered. Some branches distinguish only within drafting accuracy. Steady states are linearly stable (solid line) or unstable (dashed line).
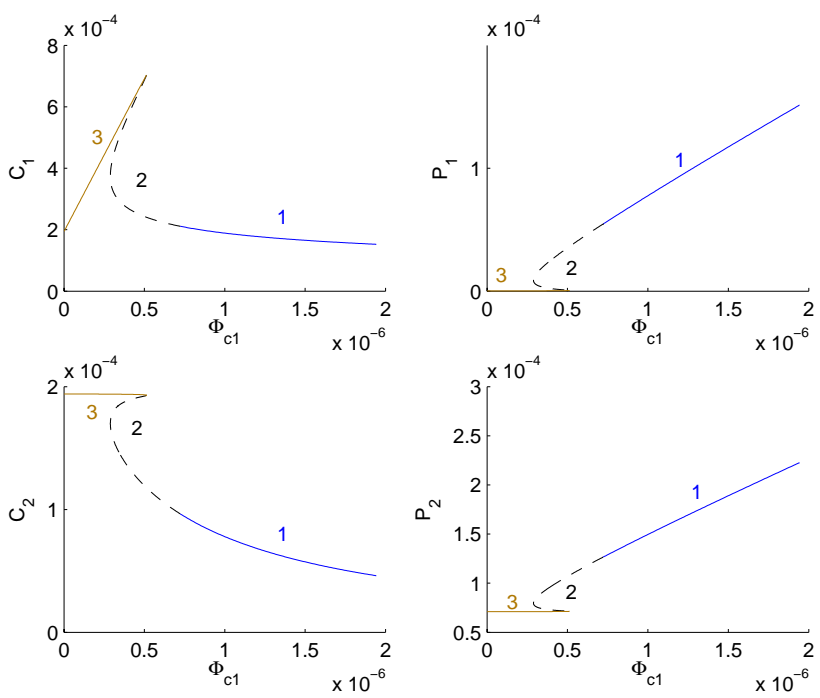

Fig. 7. As Fig. 6, but for $\Phi_{p 1}=10^{-9} s^{-1}, \Phi_{p 2}=10^{-9} s^{-1}$.

\subsection{Variation of the parameter vertical velocity $w$}

Besides the external source rates $\Phi$ the dynamics of the cloud system also strongly depends on the parameter vertical velocity $w$. Vertical velocity controls the internal interaction between cloud water concentrations of the two layers as well as the outflow of cloud water to the exterior of the system under consideration. We had already found in Sect. 3.2 for $\Phi_{p 1,2}=0$ that a steady state characterized by vanishing precipitation species $\left(P_{1,2 s}=0\right)$ is admissible provided that $w>0$, while such a state is not admissible for $w \leq 0$.
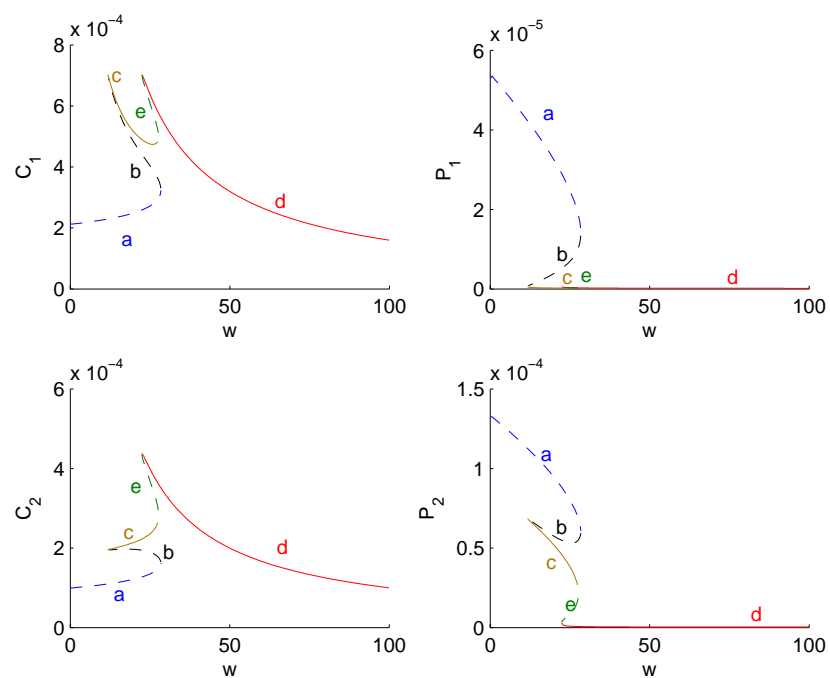

Fig. 8. Bifurcation diagram for steady states of $C_{1}, P_{1}, C_{2}$, and $P_{2}$ as functions of varying parameter upwind velocity $w$ for $\Phi_{c 1}=6 \times 10^{-7} \mathrm{~s}^{-1}, \Phi_{c 2}=10^{-6} \mathrm{~s}^{-1}, \Phi_{p 1}=10^{-9} \mathrm{~s}^{-1}, \Phi_{p 2}=10^{-9}$ $\mathrm{s}^{-1}$. Steady states are linearly stable (solid line) or unstable (dashed line).

To elucidate the role of advection for the dynamic structure of the system, the bifurcation diagram with respect to parameter $w$ is shown in Fig. 8. For upwind velocities between about 12 and $30 \mathrm{~cm} \mathrm{~s}^{-1}$ multiple steady states exist, while for weaker $w$-values the steady state is unique and unstable. A synoptic view on Figs. 7 and 8 shows that for the set of parameter values which are common to both (e.g. $w=10 \mathrm{~cm}$ $\left.\mathrm{s}^{-1}, \Phi_{c 1}=6 \times 10^{-7} \mathrm{~s}^{-1}\right)$ the single steady state is found along branches 2 and "a", respectively. For stronger upwind the transport of cloud water becomes so efficient that only few condensate is left for the growth of precipitating particles due to riming. Indeed, in the single steady state, that is the one on branch "d", precipitation concentrations are low and the cloud water contents $C_{1}$ and $C_{2}$ decrease with increasing $w$. This configuration shows some similarities to the steady state given in Eq. (11) for the limiting case $\Phi_{p 1,2}=0$ with $P_{1,2 s}=0$ and $C_{1,2 s} \propto w^{-1}$. On the other hand, for weak upwind the type of evolution is primarily determined by the external $\Phi$-sources, while advection becomes unimportant. This situation is similar to that for $w=0$ discussed in Sect. 2 . The values of the $\Phi$ 's dominate the steady state and its stability. For the selected parameter values for Fig. 8 the steady state turns out to be unstable and the attractor is a limit cycle. A range of intermediate $w$-values exists for which none of these regimes dominate. Instead the dynamics allows for multiple steady states. Two of them merge into the weakupwind regime (branch "a") and the "strong"-upwind regime (branch "d"), respectively. (Note that $w$ of the order of several tens of centimeters per second is not really considered as a strong upwind.) 
Empirical data for a systematic verification of the bifurcation diagrams of the conceptual model unfortunately are not available. Instead, the characteristica of two cloud types will be discussed with regard to the steady state types of branches "a" and "d" in Fig. 8.

A typical nimbostratus cloud is characterized by an incloud vertical air motion less than a few tens of centimeters per second and fallout of precipitation (Houze, 1993). These features are similar to the steady states described by branch "a", when disregarding their (in-)stability.

An example for a cloud which forms at moderate upwind of the order of $1 \mathrm{~m} / \mathrm{s}$ is a cirrus uncinus. According to the empirical model outlined by Houze (1993), the condensate particles are carried upwards up to the top of the updraft. In this "head" of the cloud the particles are, due to the strong vertical shear of horizontal wind, advected out of the upwind region. Downstream they move downward due to downdrafts and form the characteristic fallstreaks. The upward transport of condensate in the head is sufficiently fast to suppress precipitation formation. This situation is similar to the steady state situation according to branch "d" in Fig. 8.

\section{Discussion}

The zero-dimensional conceptional model for a cloud system, as proposed in Wacker $(1992,1998)$, is extended to a one-dimensional model to account for the vertical structure of the condensate variables and for the coupling of the dynamics in different layers due to mass transfer by advection and by sedimentation. A two-layer model is proposed, the dynamics of which is mathematically described by four coupled nonlinear ordinary differential equations. In this model, interaction of the open system with its environment is due to fall out of precipitation by sedimentation, loss of cloud water by advection out of the system, and other processes, formulated by lump-sum constant source rate $\Phi$. Internal interactions are due to riming and mass transports between the two layers by (downward) sedimentation and (upward) advection of cloud water. Structural analysis of this dynamic system shows that, compared with the zero-dimensional model, additional features arise due to vertical coupling.

In case of neglected advection, the dynamics of the single layers is coupled one-sided, that is the upper layer behaves as in the zero-dimensional model while the lower layer is subject to an additional source arising from the inflow of precipitation. Hence its longterm evolution is triggered off by the longterm evolution of the upper layer. For instance, a periodic attractor for the upper layer acts as periodic forcing on the lower layer, and the entire system develops into a periodic attractor. The characteristic time scales for the evolution in each layer decide, in which way the oscillations of the upper and lower layer variables are synchronized or whether the dynamics in the lower layer shows a superposition of varia- tions at two frequencies, that is an evolution on two different time scales (see Fig. 2).

When advection of cloud condensate by an upwind is additionally included in the model, then the dynamics in both layers is mutually coupled. Depending on the chosen values of external parameters, multiple steady states, namely up to four in the case of vanishing constant external sources $\Phi$, are found, some of which are linearly stable and some of which are linearly unstable. In particular, when varying the control parameter upwind velocity for otherwise constant parameters, three regimes are distinguished: (i) For weak upwind, the system has a unique steady state, which turns out to be unstable, and the dynamics is characterized by a periodic attractor. The constant external sources influence the evolution so that condensate concentration never remains permanently low. (ii) For "strong" upwind, the long-term behaviour is controlled by the advection of cloud water. Again the steady state is unique, but stable, such that the long-term evolution runs into a point attractor. In this final state, the input of cloud water due to the external source is mostly blown out of the upper layer. Since hardly any condensate is left for riming, precipitation concentrations remain low. Hence advection is the dominating process in this regime. (iii) For intermediate upwind velocities, however, multiple solution branches turn out, which merge by cusp and tangent bifurcations. Some of the steady states resemble the situation (i) with noteworthy precipitation concentrations, some of them situation (ii) with suppressed precipitation.

The study has shown that not only a nonstationary flow field can trigger off typical nonlinear features in the evolution of condensate concentrations, as shown by Palmer (1996), but that characteristic nonlinear effects are also triggered off by the interaction of condensate in adjacent vertical layers. The nonlinear system "cloud" proves itself to reveal many dynamical structures known from the theory of nonlinear systems. Coupling of the dynamics in several layers does not neccesssarily force the system to evolve into a unique attractor, but allows several evolution types, depending on the control parameters.

Acknowledgements. The author gratefully acknowledges C. Völker for permitting use of the program package BIFURK. She thanks E. Wessel for assisting in the linguistic part of the manuscript. This research was supported by Deutsche Forschungsgemeinschaft (grant WA1334/1).

Edited by: U. Feudel

Reviewed by: two referees

\section{References}

Cotton, W. R. and Anthes, R. A.: Storm and cloud dynamics, San Diego, Academic Press, pp 883, 1989.

Doms, G. and Schättler, U.: The nonhydrostatic limited-area model LM (Lokal-Modell) of DWD, Part I: Scientific documentation, Deutscher Wetterdienst (DWD), Offenbach, available 
at: http://cosmo-model.cscs.ch/public/documentationVer1.htm\ \#sc_doc, 1999.

Ebeling, W.: Strukturbildung bei irreversiblen Prozessen, Leipzig, BSB B. G. Teubner Verlagsges., pp 371, 1976.

Houze, R. A.: Cloud Dynamics, San Diego, Academic Press, Inc., pp 573, 1993.

Jiang, Q. and Smith, R. B.: Cloud timescales and orographic precipitation, J. Atmos. Sci., 60, 1543-1559, 2003.

Kessler, E.: On the distribution and continuity of water substance in atmospheric circulations, Meteor. Monogr., 10(32), 84, 1969.

Locatelli, J. D. and Hobbs, P. V.: Fall speed and masses of solid precipitation particles, J. Geophys. Res., 79, 2185-2197, 1974.

Nicolis, G. and Prigogine, I.: Self-organization in nonequilibrium systems, New York, J. Wiley \& Sons, pp 491, 1977.

Palmer, A. J.: A spectral model for turbulence and microphysics dynamics in an ice cloud, Nonlin. Processes Geophys., 3, 23-28, 1996, mboxhttp://www.nonlin-processesgeophys.net/3/23/1996/.

Rauber, R. M., Grant, L. O., Feng, D., and Snider, J. B.: The characteristics and distribution of cloud water over the mountains of Northern Colorado during wintertime storms, J. Climate Appl. Meteorol., 25, 468-488, 1986.
Reinhardt, T. and Wacker, U.: Impact of ice particle habits on simulated clouds, Geophys. Res. Lett., 31, L21106, doi:10.1029/2004GL021134, 2004.

Reinhardt, T. and Wacker, U.: A winter-time case study on the impact of ice particle habits on simulated clouds and precipitation, Atmos. Res., in press, 2006.

Spassova, T. and Nikolov, S.: A hierarchy of nonlinear multiparametric models of cloud dynamics and microphysics, Atmos. Res., 78, 93-102, 2005.

Wacker, U.: Structural stability in cloud physics using parameterized microphysics, Beitr. Phys. Atmos., 65, 231-242, 1992.

Wacker, U.: Competition of precipitation particles in a model with parameterized cloud microphysics, J. Atmos. Sci., 52, 25772589, 1995.

Wacker, U.: Qualitative Analyse von dynamischen Systemen der Wolkenmikrophysik, Berlin: Wissenschaft und Technik Verlag, pp 109, 1998. 